\title{
Sliding-Mode Formation Control for Cooperative Autonomous Mobile Robots
}

\author{
Michael Defoort, Thierry Floquet, Annemarie Kökösy, and Wilfrid Perruquetti
}

\begin{abstract}
This paper considers the control of a group of autonomous mobile robots. A coordinated control scheme based on a leader-follower approach is developed to achieve formation maneuvers. First and second order sliding-mode controllers are proposed for asymptotically stabilizing the vehicles to a timevarying desired formation. The latter controller, based on the relative motion states, eliminates the need for measurement or estimation of the leader velocity. It enables formation stabilization using a vision system carried by the followers and ensures the collision avoidance from the initial time instance. Experimental investigation has been conducted using a test bench made of three nonholonomic mobile robots in order to demonstrate the effectiveness of the proposed strategy.
\end{abstract}

Index Terms-Formation control, nonholonomic mobile robots, robust control, second-order sliding mode.

\section{INTRODUCTION}

$\mathbf{T}$ HE COORDINATED control of multiple autonomous mobile robots is becoming an important robotics research field. Indeed, there are many potential advantages of such systems over a single robot, including greater flexibility, adaptability, and robustness. Among all the topics of study in this field, this paper focuses on the formation control, which means to control a group of robots to form up and to move in specified geometrical shapes [1], [2]. Many cooperative tasks, such as transportation of large awkward objects, surveillance mapping, search, rescue, or area data acquisition, need the robots to maintain some prescribed formation when moving.

Various control strategies for mobile robot formations have been reported in the literature, including behaviorbased methods [3], [4], virtual structure techniques [2], [5], and leader-follower schemes [1], [6]-[9]. Among them, the leader-follower approaches have been well recognized and

Manuscript received January 15, 2008; revised July 10, 2008. First published August 15, 2008; current version published October 31, 2008. This work was supported in part by a JSPS Postdoctoral Fellowship and in part by FEDER (European Funds of Regional Development) under the ARCIR Robocoop and the AUTORIS-TAT T31 project.

M. Defoort is with the Department of System Design Engineering, Keio University, Yokohama 223-8522, Japan (e-mail: defoort@ sum.sd.keio.ac.jp).

T. Floquet and W. Perruquetti are with the Laboratoire d'Automatique, Génie Informatique et Signal, Unité Mixte de Recherche 8146, Centre National de la Recherche Scientifique, Ecole Centrale de Lille, 59651 Villeneuve-d'Ascq, France (e-mail: thierry.floquet@ec-lille.fr; wilfrid.perruquetti@ec-lille.fr).

A. Kökösy is with the Department of Signals and Systems, Institut Supérieur de 1'Electronique et du Numérique, 59046 Lille, France, and also with the Laboratoire d'Automatique, Génie Informatique et Signal, Unité Mixte de Recherche 8146, Centre National de la Recherche Scientifique, Ecole Centrale de Lille, 59651 Villeneuve-d'Ascq, France (e-mail: annemarie. kokosy@isen.fr).

Color versions of one or more of the figures in this paper are available online at http://ieeexplore.ieee.org.

Digital Object Identifier 10.1109/TIE.2008.2002717 become the most popular approaches. The basic idea of this scheme is that one robot is selected as leader and is responsible for guiding the formation. The other robots, called followers, are required to track the position and orientation of the leader with some prescribed offsets. The advantage of using such a strategy is that specifying a single quantity (the leader's motion) directs the group behavior. Therefore, this approach is simple because a reference trajectory is clearly defined by the leader and the internal formation stability is induced by the individual vehicles' control laws.

Using the leader-follower approach, the most common formation control strategies are feedback linearization [1], [6], dynamic feedback linearization [10], backstepping [11], and first order sliding-mode control [12]. In [1] and [6], the absolute velocity of the leader is treated as an exogenous input for the controller. However, in practice, it cannot be directly measured by local sensors carried by the follower. In [9], the authors proposed a formation control approach using motion segmentation and visual servoing techniques. Thus, the problem of distributed formation control in the configuration space is translated into separate visual servoing tasks in the image plane of a centralpanoramic camera. Then, the motion of the leader is estimated by the follower through the comparison of the optical flows of two pixels. In [12], the control law requires the absolute velocity and acceleration of the leader.

In practice, it is desired to have the minimum number of communication links between robots. Thus, the global motion states may not be available in some environments because there are no suitable global motion sensors. Lack of sufficient information may cause several problems such as deterioration of the overall control performance, inability to avoid collision, etc. Hence, some methods based on nonlinear observers have been recently investigated in order to estimate the global motion states: the extended Kalman filter [13], the unscented Kalman filter [10], and high gain observer [14]. Although the resulting controllers behave closely to the original one (i.e., with available global motion states) after elapsing the transient time, they suffer from the following practical drawback: some undesirable incidents, such as collision, may happen during the transient time due to the overshoots.

In this paper, a new sliding-mode formation controller which is only based on the relative motion states is derived. It eliminates the need for measurement or estimation of the absolute velocity of the leader and enables formation control using vision systems carried by the followers. Motivated by the possibility of collision avoidance between robots during the whole movement, an integral sliding-mode control strategy is proposed. Indeed, in the conventional sliding-mode control, there 


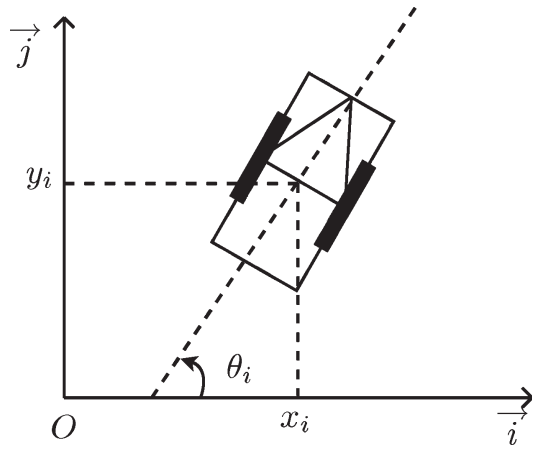

Fig. 1. Nonholonomic differentially driven mobile robot.

is a reaching phase (the transient period until the controlled system reaches the sliding surface from the initial state). During this transient period, the controlled system may be sensitive to parameter variations and disturbances because the sliding mode is not achieved. To overcome the reaching phase problems, the integral sliding-mode principle, introduced in [15], will be used.

The outline of this paper is as follows. Section II describes the dynamic model of a single robot. The coordinated formation control scheme is formulated in Section III. In Section IV, two sliding-mode formation tracking controllers are designed. The first one, based on first order sliding-mode control principle, requires total knowledge of the state variables. The second one is a robust second order sliding-mode controller. It eliminates the need of intervehicle communication, increasing the reliability of the overall system while avoiding collision between robots. Finally, in Section V, experimental results illustrate the effectiveness of the proposed strategy.

\section{Mathematical Model}

Consider a multirobot system composed of $N$ wheeled mobile robots. Each mobile robot, see Fig. 1, is of unicycle type with two driving wheels mounted on the same axis and independently controlled by two actuators (dc motors). The $i$ th robot is fully described by a 3-D vector of generalized coordinates $\mathbf{q}_{i}$ constituted by the coordinates $\left(x_{i}, y_{i}\right)$ of the midpoint between the two driving wheels and by the orientation angle $\theta_{i}$ with respect to a fixed frame

$$
\mathbf{q}_{i}=\left[x_{i}, y_{i}, \theta_{i}\right]^{\mathrm{T}} .
$$

Under the hypothesis of pure rolling and nonslipping condition, the vehicle satisfies the nonholonomic constraint

$$
\left[\begin{array}{lll}
-\sin \theta_{i} & \cos \theta_{i} & 0
\end{array}\right] \dot{\mathbf{q}}_{i}=0
$$

and the ideal kinematic equations are as follows:

$$
\dot{\mathbf{q}}_{i}=\left[\begin{array}{cc}
\cos \theta_{i} & 0 \\
\sin \theta_{i} & 0 \\
0 & 1
\end{array}\right]\left[\begin{array}{c}
w_{l, i} \\
w_{a, i}
\end{array}\right]
$$

where $w_{l, i}$ and $w_{a, i}$ are the linear and angular velocities, respectively.

However, in practical applications, the robots operate under uncertainty conditions (parameter variations, unmodeled dy- namics, etc.). Taking into account the independent actuators of the wheels and the uncertainties yields the following uncertain dynamical model describing the motion of the $i$ th robot (see [16] for further details):

$$
\begin{aligned}
{\left[\begin{array}{c}
\ddot{x}_{i} \\
\ddot{y}_{i} \\
\ddot{\theta}_{i}
\end{array}\right]=\left[\begin{array}{c}
-\dot{y}_{i} \dot{\theta}_{i} \\
\dot{x}_{i} \dot{\theta}_{i} \\
0
\end{array}\right]+} & +\left[\begin{array}{cc}
\cos \theta_{i} & 0 \\
\sin \theta_{i} & 0 \\
0 & 1
\end{array}\right] \mathbf{u}_{i} \\
& +\left[\begin{array}{cc}
\cos \theta_{i} & 0 \\
\sin \theta_{i} & 0 \\
0 & 1
\end{array}\right] \Delta_{i} \mathbf{u}_{i}+\boldsymbol{\pi}_{i}\left(\mathbf{q}_{i}, \dot{\mathbf{q}}_{i}\right) .
\end{aligned}
$$

The control input is $\mathbf{u}_{i}$

$$
\mathbf{u}_{i}=\left[\begin{array}{l}
u_{1, i} \\
u_{2, i}
\end{array}\right]=\left[\begin{array}{c}
\frac{F_{i}}{m_{i}} \\
\frac{\tau_{i}}{J_{i}}
\end{array}\right]
$$

where $m_{i}$ and $J_{i}$ are the known nominal robot mass and moment of inertia, respectively. $F_{i}$ and $\tau_{i}$ denote the force and the torque applied to the robot $i$, respectively. The disturbances (for instance, the slipping or skidding effects) are $\boldsymbol{\pi}_{i}\left(\mathbf{q}_{i}, \dot{\mathbf{q}}_{i}\right)$, and the parameter variations are as follows:

$$
\Delta_{i}=\left[\begin{array}{cc}
\varepsilon_{i} & 0 \\
0 & \varepsilon_{i}^{\prime}
\end{array}\right]
$$

where $\varepsilon_{i}$ and $\varepsilon_{i}^{\prime}$ represent the variations on the mass and the inertia of the vehicle, respectively.

Assumption 1: It is assumed that $\left\|\boldsymbol{\pi}_{i}\left(\mathbf{q}_{i}, \dot{\mathbf{q}}_{i}\right)\right\|$ is bounded by a known positive nonlinear function $\Pi_{i}\left(\mathbf{q}_{i}, \dot{\mathbf{q}}_{i}\right)$, i.e.,

$$
\left\|\boldsymbol{\pi}_{i}\left(\mathbf{q}_{i}, \dot{\mathbf{q}}_{i}\right)\right\| \leq \boldsymbol{\Pi}_{i}\left(\mathbf{q}_{i}, \dot{\mathbf{q}}_{i}\right)
$$

and that the parameter variations satisfy

$$
\left|\varepsilon_{i}\right|<1 \quad\left|\varepsilon_{i}^{\prime}\right|<1 .
$$

Remark 1: The disturbances do not necessarily satisfy the matching condition and are not assumed to be vanishing.

\section{CoOrdinated Formation CONTROL Scheme}

To achieve the coordinated formation control, it is necessary to describe the relationship, as well as the organization between the robots. Among the different ways of constructing a formation, the leader-follower approach is one of the most important building blocks. A multilayer high level formation can be realized by the combination of a first level called formation's leader and the cascades of several leader-follower pairs.

\section{A. Leader-Follower Scheme}

As shown in Fig. 2, the robot $k$ follows its leader $i$. Let $l_{i k} \in$ $\mathbb{R}_{>0}$ be the relative Euclidean distance between the robots $i$ and $k$ defined as

$$
l_{i k}=\sqrt{\left(x_{i}-x_{k}-d \cos \theta_{k}\right)^{2}+\left(y_{i}-y_{k}-d \sin \theta_{k}\right)^{2}}
$$

where $d$ is the distance between the middle point of the rear axle and the front of the robot. The coordinates $\left(x_{k}+d \cos \theta_{k}, y_{k}+\right.$ 


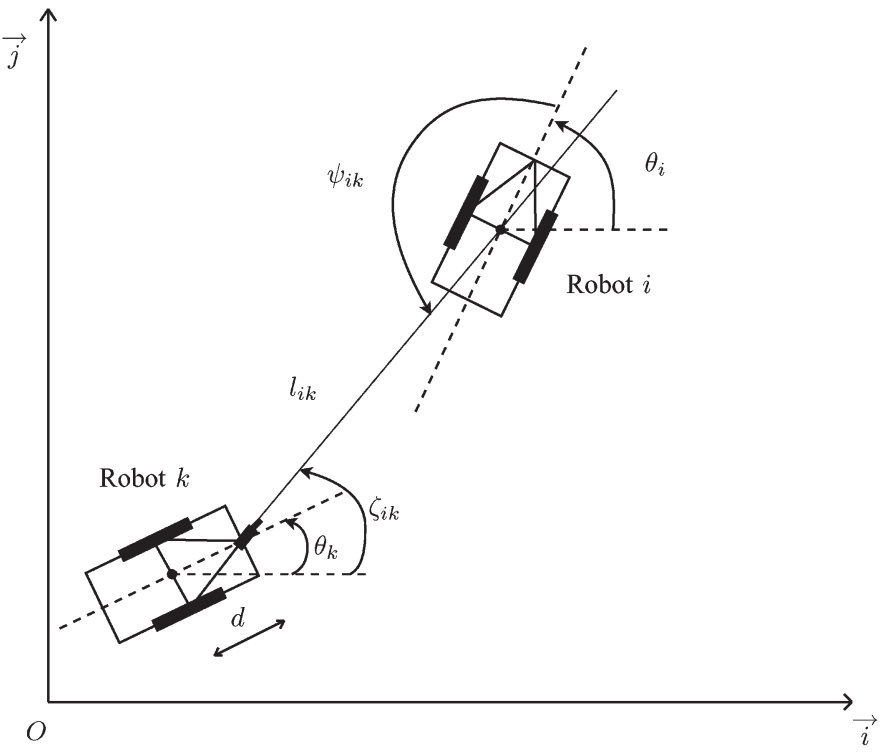

Fig. 2. Leader-follower pair.

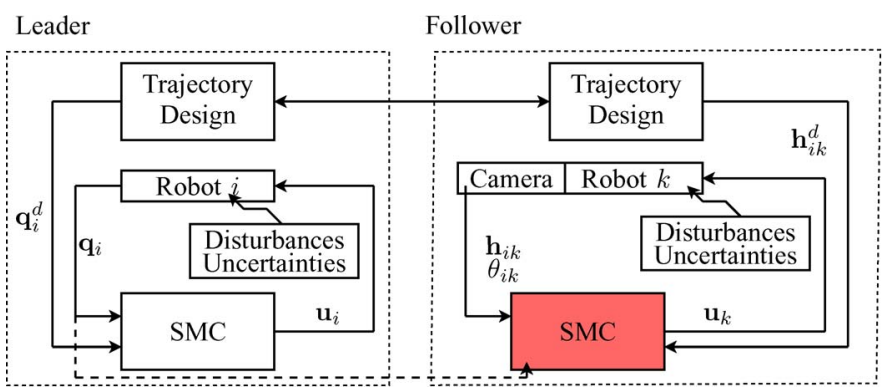

Fig. 3. Control scheme based on a leader-follower approach.

$\left.d \sin \theta_{k}\right)$ denote the position of the camera of the robot $k$. The relative bearing $\psi_{i k} \in(-\pi, \pi]$ is defined as

$$
\psi_{i k}=\pi+\zeta_{i k}-\theta_{i}
$$

and $\zeta_{i k}=\arctan \left(y_{i}-y_{k}-d \sin \theta_{k}\right) /\left(x_{i}-x_{k}-d \cos \theta_{k}\right)$.

Remark 2: If the robot $k$ is equipped with a pan-controlled monochromatic camera, the relative coordinates $l_{i k}$ and $\psi_{i k}$ can be estimated from a single image (see, for instance, [14]).

The coordination scheme is based on the relative distance and bearing between a robot $k$ and its leader $i$ (see Fig. 3). Let $\mathbf{h}_{i k}$, which is the relative configuration of the robot $k$ with respect to the robot $i$, be given by $\mathbf{h}_{i k}=\left[l_{i k}, \psi_{i k}\right]^{\mathrm{T}}$.

Differentiating twice the $l_{i k}$ and $\psi_{i k}$ yields the following input-output equations:

$$
\ddot{\mathbf{h}}_{i k}=G\left(I_{2}+\Delta_{k}\right) \boldsymbol{u}_{k}+F+P
$$

where

$$
G=\left[\begin{array}{cc}
\cos \varphi_{i k} & d \sin \varphi_{i k} \\
\frac{-\sin \varphi_{i k}}{l_{i k}} & \frac{d \cos \varphi_{i k}}{l_{i k}}
\end{array}\right]
$$

with $\varphi_{i k}=\psi_{i k}+\theta_{i k}$ and $\theta_{i k}=\theta_{i}-\theta_{k}$, and

$$
\begin{aligned}
F=\left[\dot{\psi}_{i k}^{2} l_{i k}-\dot{\theta}_{k} l_{i k} \dot{\psi}_{i k}+\dot{\theta}_{i}\left(\dot{\theta}_{i k} l_{i k}+2 \dot{\psi}_{i k} l_{i k}\right)\right. \\
\quad+\dot{\theta}_{i k}\left(\cos \left(\psi_{i k}+\theta_{i}\right) \dot{y}_{i}-\sin \left(\psi_{i k}+\theta_{i}\right) \dot{x}_{i}\right) \\
\\
\quad \frac{-2 \dot{l}_{i k} \dot{\psi}_{i k}+\dot{\theta}_{k} \dot{l}_{i k}-2 \dot{i}_{i k} \dot{\theta}_{i}-\sin \left(\psi_{i k}+\theta_{i}\right) \dot{\theta}_{i k} \dot{y}_{i}}{l_{i k}} \\
\\
\left.\quad \frac{-\cos \left(\psi_{i k}+\theta_{i}\right) \dot{\theta}_{i k} \dot{x}_{i}}{l_{i k}}\right] .
\end{aligned}
$$

$I_{2}$ denotes the $2 \times 2$ identity matrix, and $P$ reflects the disturbances and the parameter variations.

The required geometry between the leader and its follower is determined by:

- a planned trajectory $\mathbf{q}_{i}^{d}=\left(x_{i}^{d}, y_{i}^{d}, \theta_{i}^{d}\right)$ being tracked by the formation's leader. For a unicycle-type mobile robot, a saturated robust controller has been recently proposed in [17] to asymptotically stabilize the tracking errors $x_{i}^{d}-$ $x_{i}, y_{i}^{d}-y_{i}$ and $\theta_{i}^{d}-\theta_{i}$ in spite of the uncertainties.

- the desired, possibly time-varying, relative distance $l_{i k}^{d}$ and angle $\psi_{i k}^{d}$ between each leader-follower pair.

Remark 3: The following results can be extended to the two leaders scheme (i.e., $l-l$ scheme [1]) which is based on the relative distances between a follower robot $k$ and its leaders $i$ and $j$. In this case, the relative configuration of the robot $k$ is given by $\widetilde{\mathbf{h}}_{i k}=\left[l_{i k}, l_{j k}\right]^{\mathrm{T}}$. Differentiating twice $\widetilde{\mathbf{h}}_{i k}$ yields an equation similar to (8). Nevertheless, the configuration $\widetilde{\mathbf{h}}_{i k}$ is computationally more difficult to estimate than $\mathbf{h}_{i k}$ [14].

\section{B. Control Objective}

It is aimed to design a robust control law $\mathbf{u}_{k}$ such that the robot $k$ tracks its leader $i$ with a desired relative configuration $\mathbf{h}_{i k}^{d}=\left[l_{i k}^{d}, \psi_{i k}^{d}\right]^{\mathrm{T}}$, in spite of parameter uncertainties and disturbances. Furthermore, in order to prevent collisions between robots from the initial time instance, it is required that the following constraint is fulfilled.

Constraint 1: The closed-loop trajectories in (8) remains, in spite of the uncertainties, within a set of the form

$$
\Omega_{i k}=\left\{\mathbf{h}_{i k} \in \mathbb{R}^{2}:\left|l_{i k}^{d}-l_{i k}\right|<\alpha_{i k},\left|\psi_{i k}^{d}-\psi_{i k}\right|<\beta_{i k}\right\}
$$

where $\alpha_{i k}$ and $\beta_{i k}$ are suitable strictly positive parameters. This requirement ensures that $l_{i k}$ is bounded away from zero. Because $l_{i k}>0$ and $d>0$, the matrix $G$ is nonsingular.

Remark 4: Constraint 1 ensures the practical stabilization of the tracking error between the actual and the planned trajectories within a given accuracy for each follower. The trajectories of all the leaders of the formation are designed by the motion planners in such a way that the formation achieves some group objective, like, in particular, the navigation without collision between any robot in an environment with obstacles (the accuracy of the tracking error between the leader and its follower is taken into account when defining, in the motion planners, the safety 
distance to avoid collisions between robots). For further details, one can refer to [18].

Define the tracking error vector $\mathbf{e}_{i k} \in \mathbb{R}^{4}$ as

$$
\mathbf{e}_{i k}=\left[\begin{array}{c}
\mathbf{e}_{i k, 1}^{\mathrm{T}} \\
\mathbf{e}_{i k, 2}^{\mathrm{T}}
\end{array}\right]
$$

where $\mathbf{e}_{i k, 1}$ and $\mathbf{e}_{i k, 2} \in \mathbb{R}^{2}$ are defined by

$$
\left\{\begin{array}{l}
\mathbf{e}_{i k, 1}=\mathbf{h}_{i k}^{d}-\mathbf{h}_{i k} \\
\mathbf{e}_{i k, 2}=\dot{\mathbf{h}}_{i k}^{d}-\dot{\mathbf{h}}_{i k} .
\end{array}\right.
$$

The tracking error dynamics is given by

$$
\dot{\mathbf{e}}_{i k}=\left[\begin{array}{c}
\mathbf{e}_{i k, 2} \\
\ddot{\mathbf{h}}_{i k}^{d}-G\left(I_{2}+\Delta_{k}\right) \boldsymbol{u}_{k}-F-P
\end{array}\right] .
$$

Control objective: Given $l_{i k}^{d}$, $\psi_{i k}^{d}$, and the set $\Omega_{i k}$, find a controller and the corresponding compact time invariant set $\Lambda_{i k} \subset \Omega_{i k}$ (i.e., such that all trajectories $\mathbf{h}_{i k}$ of the closedloop system in (8), which starts from $\mathbf{h}_{i k}(0) \in \Lambda_{i k}$, satisfy $\mathbf{h}_{i k} \in \Lambda_{i k}$ for all $t \geq 0$ ), leading to the asymptotic stabilization of the tracking errors $\mathbf{e}_{i k}$.

\section{Controller Design}

The stabilization of the tracking errors will be achieved by using sliding-mode control laws. The aim of sliding-mode control is to constrain the system trajectories to reach and stay, after a finite time, on a given sliding manifold $\mathbf{S}_{k}^{r}$ in the state space [19]. The sliding manifold is defined by the vanishing of a corresponding sliding variable $\mathbf{s}_{k}$ and its successive time derivatives up to a certain order, i.e., the $r$ th-order sliding set

$$
\mathbf{S}_{k}^{r}=\left\{\mathbf{e}_{i k}: \mathbf{s}_{k}=\dot{\mathbf{s}}_{k}=\cdots=\mathbf{s}_{k}^{(r-1)}=0\right\} .
$$

A control law leading to such behavior is called an $r$ th order ideal sliding-mode algorithm with respect to $\mathbf{s}_{k}$. Higher order sliding modes are characterized by a discontinuous control acting on the higher order time derivatives of the sliding constraint (instead of the first time derivative in first order sliding mode). Preserving the main advantages of the former approach (relative simplicity of design and robustness properties), they can reduce the well-known chattering phenomenon, which consists in large oscillations in the neighborhood of the sliding manifold. Indeed, if the sliding order is higher than the relative degree of the system, the discontinuity induced by the variable structure control law is embedded in the higher order time derivatives of the sliding constraint instead of the first time derivative in classical sliding mode. Furthermore, they guarantee better convergence accuracy (see [20] for a survey).

In order to fulfill Constraint 1, it is necessary that the state $\mathbf{h}_{i k}$ is constrained to asymptotically track $\mathbf{h}_{i k}^{d}$ with some prescribed dynamics in spite of the uncertainties. To achieve this goal, the reaching phase must be removed because the output behavior, during this phase, cannot be predetermined by the sliding surface. Hence, the sliding variable is designed in order to eliminate the reaching phase. The sliding-mode control algorithm is designed in two steps: 1) the selection of a suitable sliding variable $\mathbf{s}_{k}$ such that, while sliding, the control objective is fulfilled and 2) the design of the corresponding control input $\mathbf{u}_{k}$ constraining the system trajectories to evolve on the sliding surface from the initial time instance.

This design can be made using two levels of information.

1) The first one, based on first-order sliding-mode control, requires the knowledge of the velocity $\dot{\mathbf{q}}_{i}$ of the robot $i$, which is quite complex from the point of view of information flow. It also requires the velocity $\dot{\mathbf{q}}_{k}$ of the robot $k$.

2) The second one, based on second-order sliding-mode control, is simpler from the information flow point of view and does not require information about the velocities of the robots $i$ and $k$.

\section{A. First Order Sliding-Mode Controller $(r=1)$}

In this part, the control objective is to generate a first order sliding mode on a sliding surface appropriately chosen for each follower, that is to say to constrain the system trajectories to evolve from the initial time instance on $\mathbf{S}_{k}^{1}=\left\{\mathbf{e}_{i k}: \mathbf{s}_{k}=0\right\}$.

Assumption 2: It is assumed that each robot $k$ knows the following: 1) its relative position $\left.\left[l_{i k}, \psi_{i k}\right] ; 2\right)$ its relative orientation $\theta_{i k} ; 3$ ) its velocity $\dot{\mathbf{q}}_{k} ; 4$ ) the angle $\theta_{i}$ of the leader $i$; and 5) the velocity $\dot{\mathbf{q}}_{i}$ of the leader $i$.

The time derivatives $\dot{l}_{i k}$ and $\dot{\psi}_{i k}$ of the relative position can be computed because $l_{i k}, \psi_{i k}, \theta_{i k}, \theta_{i}, \dot{\mathbf{q}}_{i}$, and $\dot{\mathbf{q}}_{k}$ are known. Therefore, the values of $G$ and $F$ can be computed.

Assumption 3: It is supposed that, within the set $\Omega_{i k}$, the following parameter uncertainties are bounded as follows:

$$
\left\|G \Delta_{k} G^{-1}\right\| \leq 1-c_{k}
$$

with $0<c_{k}<1$.

Consider the following feedback applied to system (12):

$$
\boldsymbol{u}_{k}=-G^{-1}\left(-\ddot{\mathbf{h}}_{i k}^{d}+F+\mathbf{v}_{k}\right)
$$

where $\mathbf{v}_{k} \in \mathbb{R}^{2}$ is the new control input. Thus, the control objective becomes, to asymptotically stabilize, the uncertain "double integrator" system

$$
\begin{aligned}
\dot{\mathbf{e}}_{i k}=\left[\begin{array}{ll}
0_{2} & I_{2} \\
0_{2} & 0_{2}
\end{array}\right] & \mathbf{e}_{i k}+\left[\begin{array}{l}
0_{2} \\
I_{2}
\end{array}\right]\left[\left(I_{2}+G \Delta_{k} G^{-1}\right) \mathbf{v}_{k}\right] \\
& +\left[\begin{array}{c}
0_{2} \\
I_{2}
\end{array}\right]\left[-P+G \Delta_{k} G^{-1}\left(F-\ddot{\mathbf{h}}_{i k}^{d}\right)\right]
\end{aligned}
$$

where $0_{2}$ denotes the $2 \times 2$ zero matrix.

Let us define the sliding variable as

$$
\mathbf{s}_{k}(t)=\mathbf{e}_{i k, 2}(t)+K_{k} \mathbf{e}_{i k, 1}(t)+L_{k} \mathbf{d}_{k}(t)
$$

with

$$
K_{k}=\left[\begin{array}{cc}
K_{1, k} & 0 \\
0 & K_{2, k}
\end{array}\right] \quad L_{k}=\left[\begin{array}{cc}
L_{1, k} & 0 \\
0 & L_{2, k}
\end{array}\right] .
$$

$K_{1, k}, K_{2, k}, L_{1, k}$, and $L_{2, k}$ are strictly positive constants that will be defined later. The term $\mathbf{d}_{k} \in \mathbb{R}^{2}$ induces the integral 
term and provides one more degree of freedom in the sliding variable design. Its dynamics fulfill the differential equation

$$
\left\{\begin{array}{l}
\dot{\mathbf{d}}_{k}(t)=\mathbf{e}_{i k, 1}(t) \\
\mathbf{d}_{k}(0)=L_{k}^{-1}\left(-\mathbf{e}_{i k, 2}(0)-K_{k} \mathbf{e}_{i k, 1}(0)\right) .
\end{array}\right.
$$

The control law $\mathbf{v}_{k}$ is designed as follows:

$$
\mathbf{v}_{k}=-K_{k} \mathbf{e}_{i k, 2}-L_{k} \mathbf{e}_{i k, 1}+\Delta \mathbf{v}_{k}
$$

where $\Delta \mathbf{v}_{k}$ represents the sliding-mode control part that is designed to reject the perturbations and is given by

$$
\Delta \mathbf{v}_{k}=-\lambda_{k} \operatorname{Sign}\left(\mathbf{s}_{k}\right) .
$$

The signum function is defined as $\operatorname{Sign}\left(\left[s_{1, k}, s_{2, k}\right]^{\mathrm{T}}\right)=$ $\left[\operatorname{sign}\left(s_{1, k}\right), \operatorname{sign}\left(s_{2, k}\right)\right]^{\mathrm{T}}$. The gain $\lambda_{k}$ is expressed as

$$
\lambda_{k}=\frac{\rho_{k}+\eta}{c_{k}}
$$

with the constant $\eta>0$ and the known function $\rho_{k} \geq \max _{\left|\varepsilon_{k}\right|,\left|\varepsilon_{k}^{\prime}\right|} \| G \Delta_{k} G^{-1}\left(F-\ddot{\mathbf{h}}_{i k}^{d}+K_{k} \mathbf{e}_{i k, 2}+\right.$ $\left.L_{k} \mathbf{e}_{i k, 1}\right)\left\|+\max _{\left\|\pi_{k}\right\| \leq \Pi_{k}}\right\| P \|$.

Theorem 1: Under Assumptions 1-3, the controller defined by (14), (18), and (19) with the sliding variable (16) and (17) ensures that the tracking errors given by (12) are asymptotically stable. Moreover, if $\mathbf{h}_{i k}(0) \in \Lambda_{i k}$, the collision avoidance between the leader and its corresponding follower is guaranteed from the initial time instance.

Proof: Let us choose the candidate Lyapunov function

$$
V_{k}=\frac{1}{2} \mathbf{s}_{k}^{\mathrm{T}} \mathbf{s}_{k}
$$

Using (15)-(20), the time derivative of $V_{k}$ along the trajectory of system (12) is expressed as

$$
\begin{aligned}
\dot{V}_{k}= & \mathbf{s}_{k}^{\mathrm{T}}\left(\left(I_{2}+G \Delta_{k} G^{-1}\right) \mathbf{v}_{k}+G \Delta_{k} G^{-1}\left(F-\ddot{\mathbf{h}}_{i k}^{d}\right)\right) \\
& +\mathbf{s}_{k}^{\mathrm{T}}\left(K_{k} \mathbf{e}_{i k, 2}+L_{k} \mathbf{e}_{i k, 1}-P\right) \\
= & -\mathbf{s}_{k}^{\mathrm{T}}\left(I_{2}+G \Delta_{k} G^{-1}\right) \lambda_{k} \operatorname{Sign}\left(\mathbf{s}_{k}\right)-\mathbf{s}_{k}^{\mathrm{T}} P \\
& +\mathbf{s}_{k}^{\mathrm{T}} G \Delta_{k} G^{-1}\left(F-\ddot{\mathbf{h}}_{i k}^{d}+K_{k} \mathbf{e}_{i k, 2}+L_{k} \mathbf{e}_{i k, 1}\right) \\
\leq & -\lambda_{k}\left\|\mathbf{s}_{k}\right\|+\left(1-c_{k}\right) \lambda_{k}\left\|\mathbf{s}_{k}\right\|+\|P\|\left\|\mathbf{s}_{k}\right\| \\
& +\left\|G \Delta_{k} G^{-1}\left(F-\ddot{\mathbf{h}}_{i k}^{d}+K_{k} \mathbf{e}_{i k, 2}+L_{k} \mathbf{e}_{i k, 1}\right)\right\|\left\|\mathbf{s}_{k}\right\| \\
\leq & -c_{k} \lambda_{k}\left\|\mathbf{s}_{k}\right\|+\rho_{k}\left\|\mathbf{s}_{k}\right\| \\
\leq & -\eta\left\|\mathbf{s}_{k}\right\| .
\end{aligned}
$$

Thus, it is guaranteed that the output trajectories reach the surface in spite of uncertainties and disturbances. Moreover, the initial condition $\mathbf{d}_{k}(0)$ is determined such that the sliding variable always satisfies $\mathbf{s}_{k}(0)=0$. Hence, the trajectory evolves on the sliding surface $\left\{\mathbf{s}_{k}=0\right\}$ from the initial time instance without any reaching phase and remains there.

The time derivative in (16) yields the sliding dynamics

$$
\dot{\mathbf{s}}_{k}=\dot{\mathbf{e}}_{i k, 2}+K_{k} \mathbf{e}_{i k, 2}+L_{k} \mathbf{e}_{i k, 1} .
$$

In sliding mode, the equivalent control $\mathbf{v}_{k}^{\mathrm{eq}}$, obtained by writing $\dot{\mathbf{s}}_{k}=0$ (see [22] for further details), is given by

$$
\begin{aligned}
\mathbf{v}_{k}^{\mathrm{eq}}=\left(I_{2}+G \Delta_{k} G^{-1}\right)^{-1}\left(-G \Delta_{k} G^{-1}\left(F-\ddot{\mathbf{h}}_{i k}^{d}\right)+P\right) \\
+\left(I_{2}+G \Delta_{k} G^{-1}\right)^{-1}\left(-K_{k} \mathbf{e}_{i k, 2}-L_{k} \mathbf{e}_{i k, 1}\right) .
\end{aligned}
$$

Substituting the previous equation $\mathbf{v}_{k}^{\text {eq }}$ into (15), one obtains the equivalent closed-loop dynamics, in sliding mode

$$
\dot{\mathbf{e}}_{i k}=\left[\begin{array}{cc}
0_{2} & I_{2} \\
-L_{k} & -K_{k}
\end{array}\right] \mathbf{e}_{i k} .
$$

Therefore, in sliding mode, the tracking errors are constrained to fulfill the following differential equations:

$$
\left\{\begin{array}{l}
\left(\ddot{l}_{i k}^{d}-\ddot{l}_{i k}\right)+K_{1, k}\left(\dot{l}_{i k}^{d}-\dot{l}_{i k}\right)+L_{1, k}\left(l_{i k}^{d}-l_{i k}\right)=0 \\
\left(\ddot{\psi}_{i k}^{d}-\ddot{\psi}_{i k}\right)+K_{2, k}\left(\dot{\psi}_{i k}^{d}-\dot{\psi}_{i k}\right)+L_{2, k}\left(\psi_{i k}^{d}-\psi_{i k}\right)=0 .
\end{array}\right.
$$

The positive constants $K_{1, k}, K_{2, k}, L_{1, k}, L_{2, k}$, and the corresponding nonempty set of initial conditions $\Lambda_{i k} \subset \Omega_{i k}$ are selected straightforwardly using pole assignment technique such that, in sliding mode, the tracking errors are exponentially stable and the set $\Lambda_{i k}$ is positively invariant for the equivalent closed-loop system (23), i.e., $\forall t \geq 0, \mathbf{h}_{i k}(t) \in \Lambda_{i k}$.

Using the control input with the proposed sliding variable, the system (15) can be controlled in sliding mode from the initial time instance. Thus, the discontinuous control term maintains the system on the sliding surface by rejecting the effect of the uncertainties for $t \geq 0$. The outputs are insensitive to the system uncertainties, and the closed-loop behavior in (12) is given by (22), which is asymptotically stable. As the transient process is removed, collision avoidance is ensured in spite of the uncertainties from the initial time instance.

\section{B. Second Order Sliding-Mode Controller $(r=2)$}

Because the proposed first order sliding-mode controller depends on $\dot{\mathbf{q}}_{i}$ and $\dot{\mathbf{q}}_{k}$, it is necessary that the robot $k$ has knowledge of its velocity and the velocity of the robot $i$. In order to minimize the information flow, a controller that only needs the formation error $\mathbf{e}_{i k, 1}$ is proposed hereafter. Its design is still based on sliding-mode control. However, as the state $\mathbf{e}_{i k, 2}$ is not available, the sliding variable (16) and (17) cannot be considered in the controller design. A second order slidingmode controller is proposed to circumvent this difficulty.

Assumption 4: It is assumed that each robot knows the following: 1) its relative position $\left.\left[l_{i k}, \psi_{i k}\right] 2\right)$ its relative orientation $\theta_{i k}$.

Therefore, $G$ can be computed. Because $F$ in the model (8) is unknown, it is treated as model uncertainty of the system.

Assumption 5: It is assumed that $\mathbf{e}_{i k, 2}(0)$ is known, that is to say $\dot{\mathbf{h}}_{i k}(0)$ is known.

Remark 5: The condition of the knowledge of $\dot{\mathbf{h}}_{i k}(0)$ is not conservative. Indeed, the initial configuration of the robot formation can be divided into two cases.

1) All the initial robot velocities are equal to zero. Then, one has $\mathbf{e}_{i k, 2}(0)=\dot{\mathbf{h}}_{i k}^{d}(0)$. 
2) The initial robot velocities are not equal to zero. In this case, before applying the proposed controller, one can design a robust finite time exact differentiator [21] in order to obtain the initial conditions $\dot{\mathbf{h}}_{i k}(0)$.

Let us define the sliding variable as follows:

$$
\overline{\mathbf{s}}_{k}=\left[\begin{array}{c}
\bar{s}_{1, k} \\
\bar{s}_{2, k}
\end{array}\right]=\mathbf{e}_{i k, 1}+K_{k} \overline{\mathbf{d}}_{k}+L_{k} \overline{\mathbf{c}}_{k}
$$

where

$$
K_{k}=\left[\begin{array}{cc}
K_{1, k} & 0 \\
0 & K_{2, k}
\end{array}\right] \quad L_{k}=\left[\begin{array}{cc}
L_{1, k} & 0 \\
0 & L_{2, k}
\end{array}\right]
$$

are strictly positive definite diagonal matrices. The functions $\overline{\mathbf{d}}_{k}$ and $\overline{\mathbf{c}}_{k}$ still induce integral terms. Their dynamics fulfill the following differential equations:

$$
\left\{\begin{array}{l}
\dot{\overline{\mathbf{d}}}_{k}=\mathbf{e}_{i k, 1} \\
\dot{\overline{\mathbf{c}}}_{k}=\overline{\mathbf{d}}_{k} \\
\overline{\mathbf{d}}_{k}(0)=L_{k}^{-1}\left(-\mathbf{e}_{i k, 2}(0)-K_{k} \mathbf{e}_{i k, 1}(0)\right) \\
\overline{\mathbf{c}}_{k}(0)=L_{k}^{-1}\left(-\mathbf{e}_{i k, 1}(0)-K_{k} \overline{\mathbf{d}}_{k}(0)\right) .
\end{array}\right.
$$

Differentiating twice the variable (24) gives

$$
\ddot{\overline{\mathbf{s}}}_{k}=K_{k} \mathbf{e}_{i k, 2}+L_{k} \mathbf{e}_{i k, 1}+\ddot{\mathbf{h}}_{i k}^{d}-G\left(I_{2}+\Delta_{k}\right) \boldsymbol{u}_{k}-F-P .
$$

Let us define the following state feedback control:

$$
\boldsymbol{u}_{k}=-G^{-1}\left(\left[\begin{array}{c}
v_{1, k} \\
v_{2, k}
\end{array}\right]-L_{k} \mathbf{e}_{i k, 1}-\ddot{\mathbf{h}}_{i k}^{d}\right)
$$

such that one gets

$$
\ddot{\overline{\mathbf{s}}}_{k}=\left(I_{2}+G \Delta_{k} G^{-1}\right)\left[\begin{array}{l}
v_{1, k} \\
v_{2, k}
\end{array}\right]-\bar{P}
$$

where

$$
\bar{P}=F+P-K_{k} \mathbf{e}_{i k, 2}+G \Delta_{k} G^{-1}\left(L_{k} \mathbf{e}_{i k, 1}+\ddot{\mathbf{h}}_{i k}^{d}\right) .
$$

Assumption 6: It is supposed that, within the set $\Omega_{i k}$, the following parameter variations are bounded as follows:

$$
0<\delta_{k} \leq\left\|I_{2}+G \Delta_{k} G^{-1}\right\| \leq \delta_{k}^{\prime} .
$$

Furthermore, one can get an upper bound of the uncertainties $\|\bar{P}\|$ within the set $\Omega_{i k}$, i.e., there are a positive constant $\bar{\rho}_{k}$ such that

$$
\|\bar{P}\| \leq \bar{\rho}_{k} .
$$

According to (28), the relative degree (see [23] for details) of system (12) with respect to the sliding variable $\overline{\mathbf{s}}_{k}$ is two. Thus, one must design a second order sliding-mode algorithm that only requires the knowledge of $\overline{\mathbf{s}}_{k}$ (that is to say, only the relative configuration). One can use the sampled twisting algorithm, described in [20], i.e., $\forall j=\{1,2\}$

$$
v_{j, k}= \begin{cases}-\mu_{k} \operatorname{sign}\left(\bar{s}_{j, k}\right), & \text { if } \bar{s}_{j, k} \Delta_{\bar{s}_{j, k}}>0 \\ -\nu_{k} \operatorname{sign}\left(\bar{s}_{j, k}\right), & \text { if } \bar{s}_{j, k} \Delta_{\bar{s}_{j, k}} \leq 0\end{cases}
$$

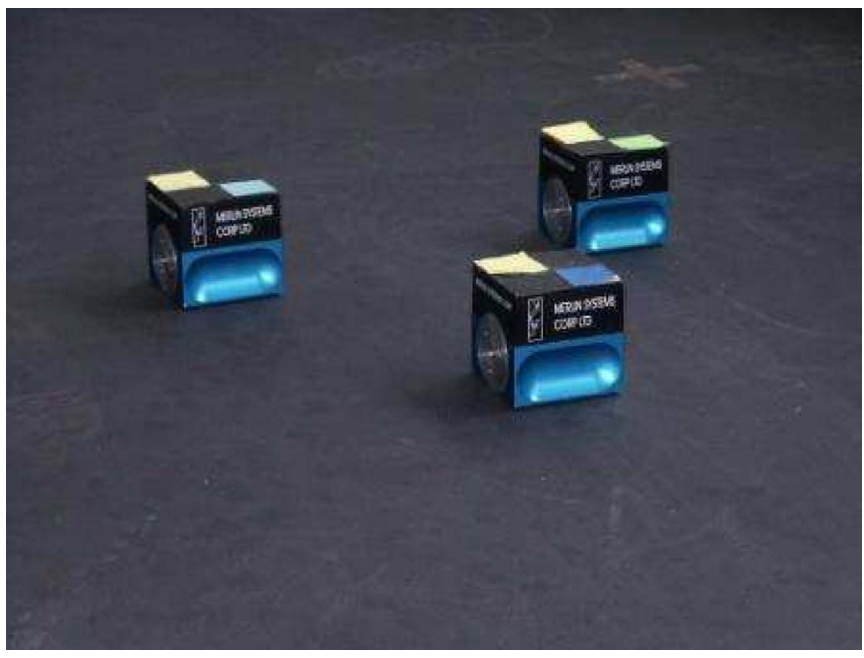

Fig. 4. Miabot robots.

TABLE I

SPECIFICATIONS OF THE ROBOT

\begin{tabular}{|l|l|}
\hline Processor: & Atmel ATMega64 \\
Name & $64 \mathrm{~Kb}$ \\
Flash Memory & $2 \mathrm{~Kb}$ \\
EEPROM & $4 \mathrm{~Kb}$ \\
SRAM & up to 14.5 MIPS \\
Speed & \\
\hline Drive train: & $6 \mathrm{~V}$ \\
Dual DC Motors & $0.04 \mathrm{~mm}$ resolution \\
Encoders & $52 \mathrm{~mm}$ \\
Wheel diameter & $8: 1$ \\
Gearbox Ratio & $3 \mathrm{~m} / \mathrm{s}$ \\
Speed & \\
\hline Batteries: & $6 \times 1.2 \mathrm{~V}$ AA NiMH cells \\
NiMH & Approx. $2 \mathrm{H}$ \\
Operation & Bluetooth \\
\hline Communications: & up yo $100 \mathrm{~m}$ \\
Wireless & Bidirectional at $11.5 \mathrm{~kb} / \mathrm{s}$ \\
Range & \\
Speed & aluminium \\
\hline Chassis: & $7.5 \mathrm{~cm}^{3}$ \\
Construction & \\
Case dimensions &
\end{tabular}

with

$$
\Delta_{\bar{s}_{j, k}}= \begin{cases}0, & \text { if } \gamma=0 \\ \bar{s}_{j, k}(\gamma \tau)-\bar{s}_{j, k}((\gamma-1) \tau), & \text { if } \gamma \geq 1\end{cases}
$$

where $\tau$ is the sampling period, and $\nu_{k}$ and $\mu_{k}$ are positive constants satisfying the following conditions:

$$
\nu_{k}>\frac{\bar{\rho}_{k}}{\delta_{k}} \quad \mu_{k}>\frac{\delta_{k}^{\prime} \nu_{k}}{\delta_{k}}+2 \frac{\bar{\rho}_{k}}{\delta_{k}} .
$$

This algorithm provides good robustness properties. It does not require the knowledge of the time derivative of the sliding variable $\overline{\mathbf{s}}_{k}$ and takes into account some practical constraints such as the sampling of the measurement and the control.

Remark 6: One can note that the suboptimal algorithm described in [24] could also be applied. After an initialization time, this algorithm needs, in real time, the exact knowledge of the singular value of the sliding variable, that is to say, the 


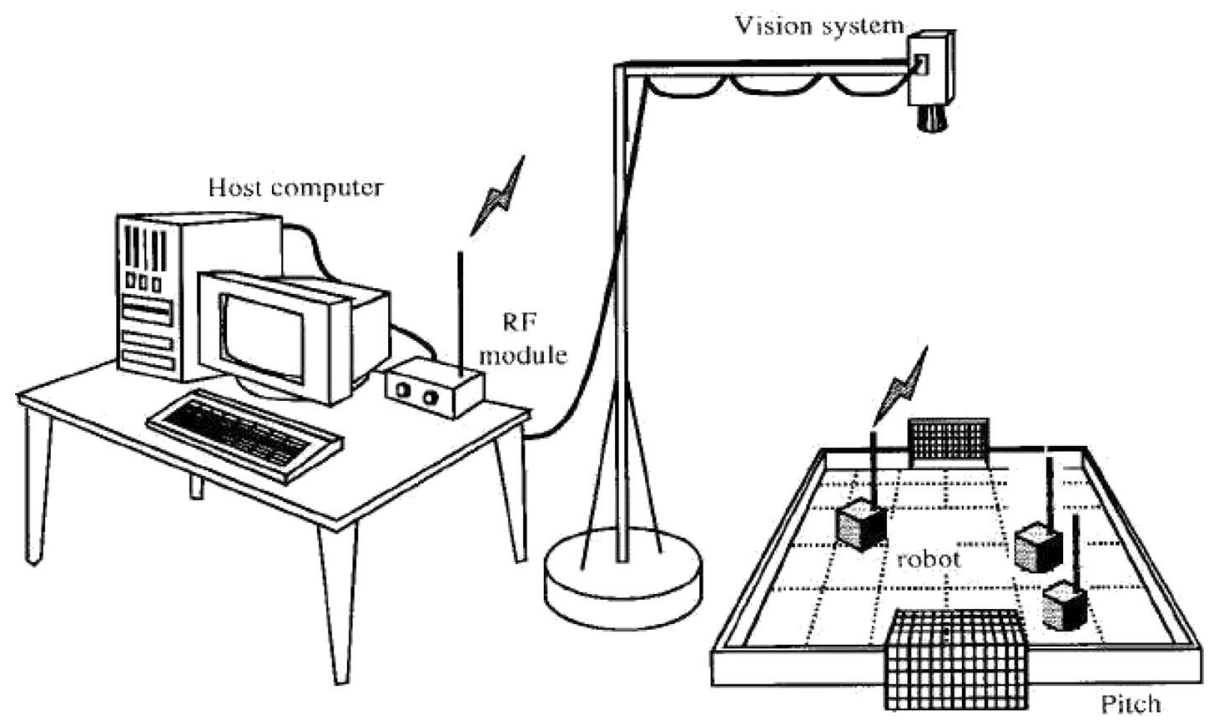

Fig. 5. Overview of the platform.

corresponding value when $\overline{\mathbf{s}}_{k}=0$. Thus, its implementation is less easy than the sample twisting algorithm.

Theorem 2: Under Assumptions 1 and 4-6, using only the relative configuration, the controller defined in (27) and (31), with the sliding variable (24) and (25), ensures that the tracking errors given by (12) are asymptotically stable. Moreover, if $\mathbf{h}_{i k}(0) \in \Lambda_{i k}$, the collision avoidance between the leader and its corresponding follower is guaranteed from the initial time instance.

Proof: The proof is quite similar to the proof of Theorem 1 and is omitted.

\section{EXPERIMENTS}

\section{A. Experimental Setup}

To demonstrate the effectiveness of the proposed second order sliding-mode controller, experiments have been conducted on a test bench which consists of three nonholonomic mobile robots, called MIABOT (see Fig. 4). The detailed specifications of a mobile robot are given in Table I.

The perspective view of the platform with the vision system, host computer, communication system, and pitch is shown in Fig. 5. The vision system consists of a TMC-7 charge-coupled device camera with a resolution of $320 \times 240$ pixels and an image grabber with a processing rate of $30 \mathrm{frames} / \mathrm{s}$. The vision system recognizes each vehicle by the color marker adhibited on the top of each one. A virtual vision sensor is fixed on the front of each follower $(d=5 \mathrm{~cm})$. It translates the relative positions obtained by the actual vision system into the relative motion states from the view of the follower. These data are transmitted to the host computer. Because the localization of the robots is made only by the vision system, its processing rate limits the sampling time for the controller. It is set to $\tau=60 \mathrm{~ms}$. In the experiments, the robots are controlled with a host computer, which is a Pentium processor with $2.4 \mathrm{GHz}$. However, it is easy to control the robots as a decentralized system because the proposed strategy is decentralized. A private IP is assigned to each robot beforehand, and mobile robots

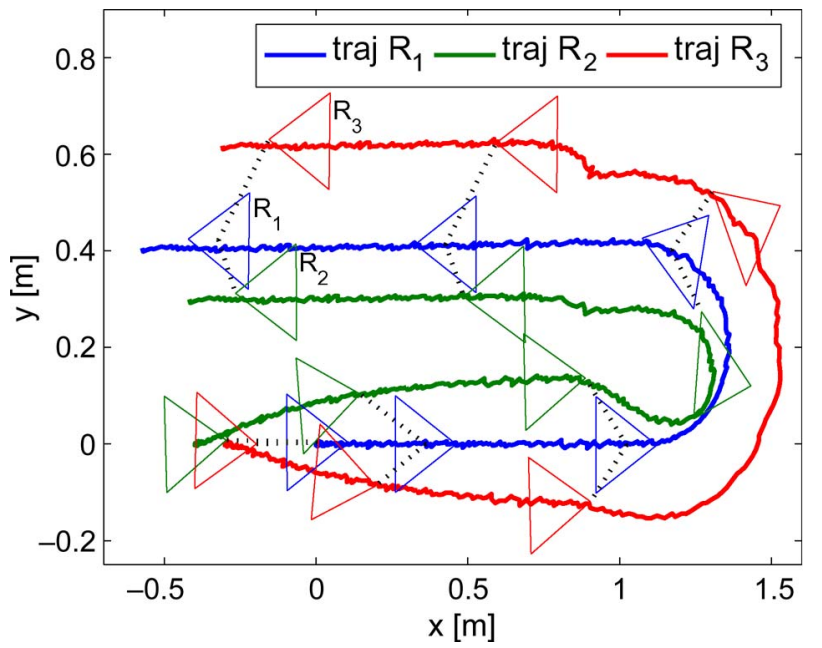

Fig. 6. Trajectories of the leader $R_{1}$ and the followers $R_{2}$ and $R_{3}$.

(clients) are connected with a server through the communication system, which is a radio frequency module.

\section{B. Experimental Results}

A scenario with one leader and two followers is considered. The objective is that the formation moves in a time-varying geometric shape characterizing by the desired parameters

$$
\begin{array}{ll}
l_{12}^{d}=0.175-0.025 \tanh (10(t-5.5)) & \psi_{12}^{d}=-\frac{\pi}{4} \\
l_{13}^{d}=0.25+0.05 \tanh (10(t-5.5)) & \psi_{13}^{d}=\frac{\pi}{4} .
\end{array}
$$

These parameters have been planned such that the desired trajectories are sufficiently smooth. They characterize a triangular formation with modification of its size at $t=5.5$ s (i.e., $l_{12}^{d}$ : $0.2 \mathrm{~m} \rightarrow_{t=5.5 \mathrm{~s}} 0.15 \mathrm{~m}$ and $\left.l_{13}^{d}: 0.2 \mathrm{~m} \rightarrow_{t=5.5 \mathrm{~s}} 0.3 \mathrm{~m}\right)$. The formation's leader is $R_{1}$. Its desired trajectory is planned using the algorithm given in [18]. It consists of two straight lines traveled with a linear velocity of $0.5 \mathrm{~m} / \mathrm{s}$ and an arc of a circle. It 


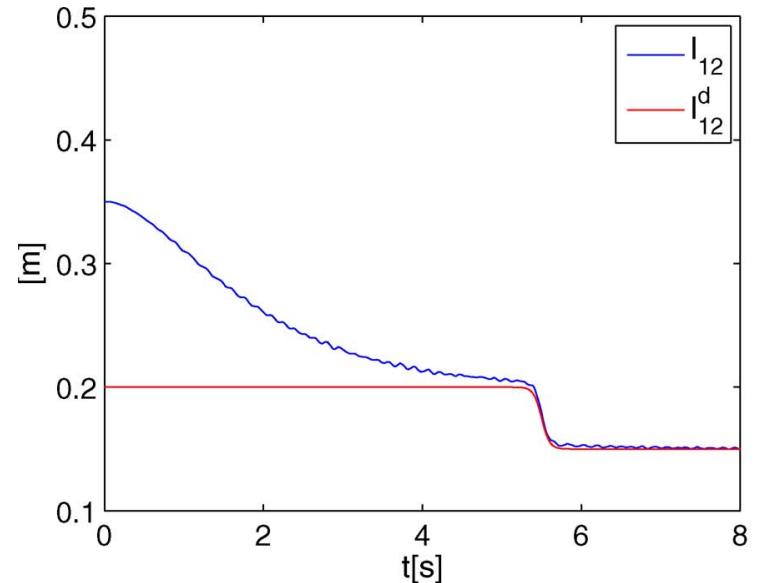

(a)

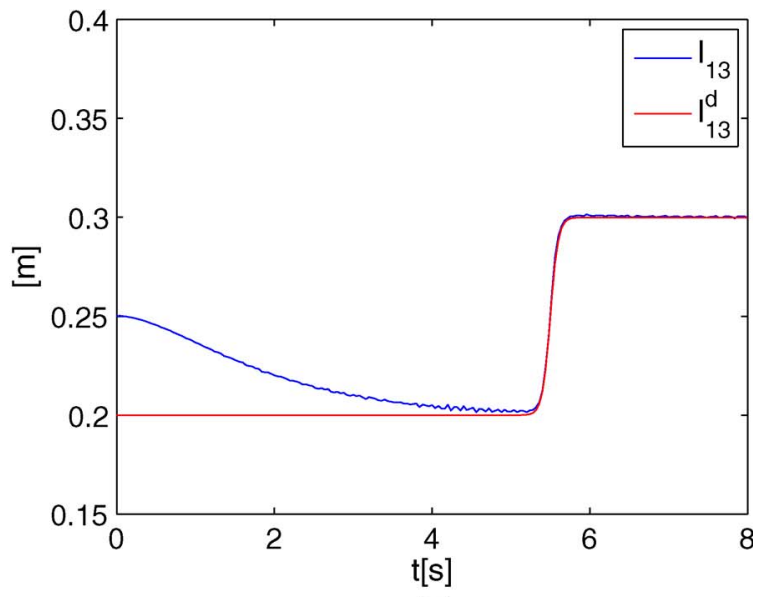

(b)

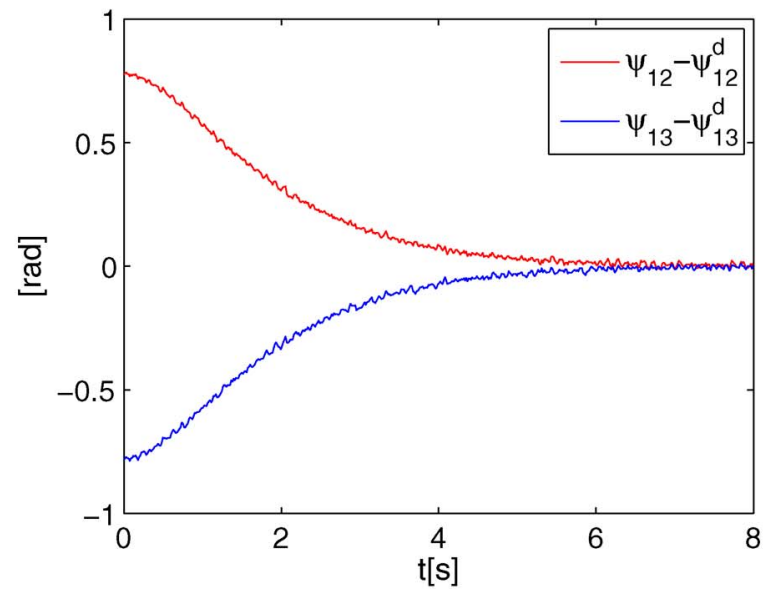

(c)

Fig. 7. Tracking errors.

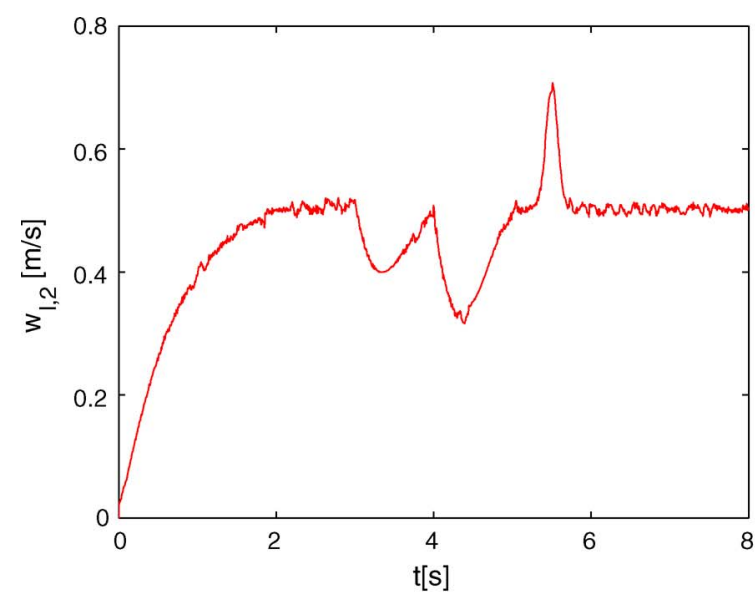

(a)

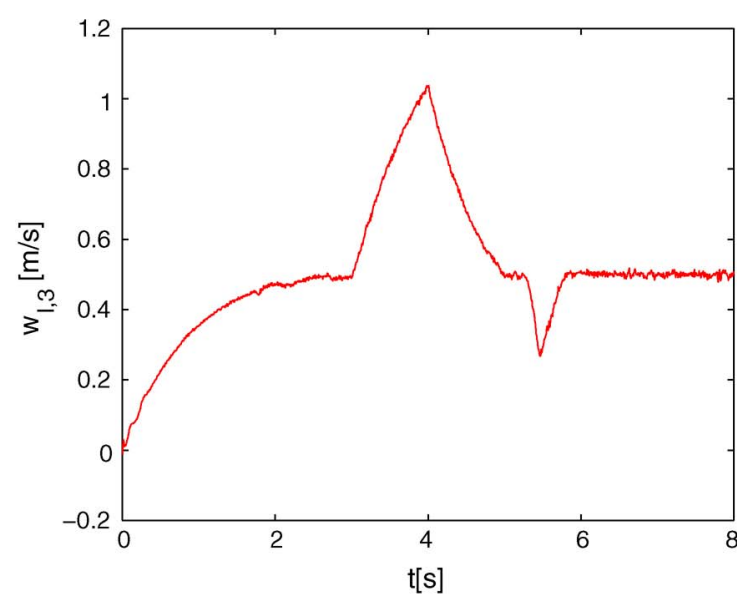

(b)

Fig. 8. Linear velocities of robots $R_{2}$ and $R_{3}$.

has been designed such that the followers have sufficient control authority to track the desired configuration and stay inside the game area. Note that the tracking of the planned trajectory by the formation's leader is achieved via first order sliding-mode control because it knows its own velocity.

The initial configurations of the robots, which characterize a linear formation, are $\mathbf{q}_{1}(0)=[0 \mathrm{~m}, 0 \mathrm{~m}, 0 \mathrm{rad}]^{\mathrm{T}}, \mathbf{q}_{2}(0)=$
$[-0.4 \mathrm{~m}, 0 \mathrm{~m}, 0 \mathrm{rad}]^{\mathrm{T}}$, and $\quad \mathbf{q}_{3}(0)=[-0.3 \mathrm{~m}, 0 \mathrm{~m}, 0 \mathrm{rad}]^{\mathrm{T}}$. Hence, the geometrical shape of the group will be reconfigured from a "linear" to a "triangular" shape. Their initial velocities are zero. The velocity of the leader $R_{1}$ is not known by the followers. Thus, the first order sliding-mode controller cannot be used. That is why, in order to design the control inputs $\mathbf{u}_{2}$ and $\mathbf{u}_{3}$, the second-order sliding-mode controller 


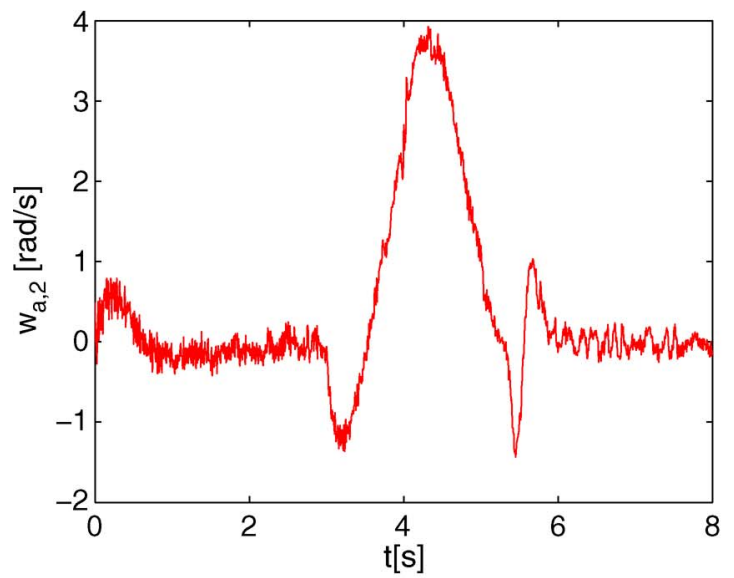

(a)

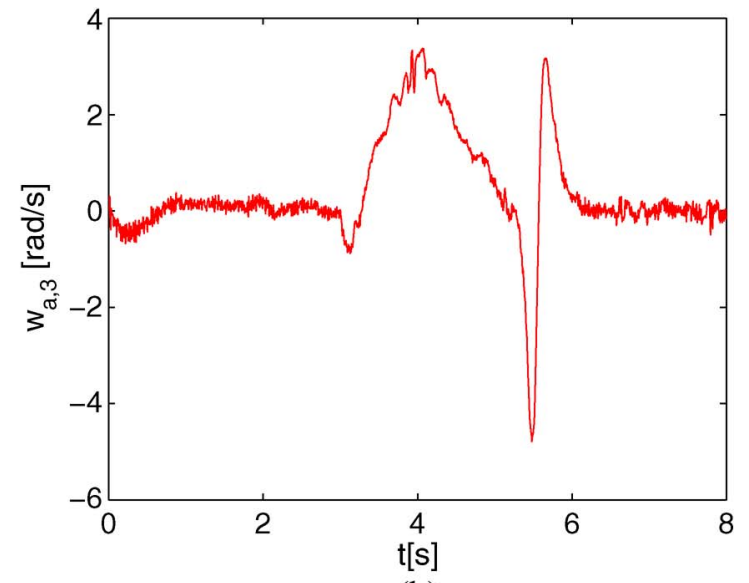

(b)

Fig. 9. Angular velocities of robots $R_{2}$ and $R_{3}$.

is applied. The parameters of the sliding variable (24) are as follows:

$$
K_{2}=K_{3}=\left[\begin{array}{ll}
2 & 0 \\
0 & 2
\end{array}\right] \quad L_{2}=L_{3}=\left[\begin{array}{ll}
1 & 0 \\
0 & 1
\end{array}\right] .
$$

The controller gains are $\nu_{2}=\nu_{3}=4$ and $\mu_{2}=\mu_{3}=20$.

Under the proposed formation controller, the trajectories of the two robots, recorded by the vision system, are shown in Fig. 6. The corresponding tracking errors for the two followers are shown in Fig. 7. One can see that they are asymptotically stable in spite of the uncertainties. The geometric shape of the formation is reconfigured in a "triangular" shape in $5 \mathrm{~s}$. Then, this triangular formation is tracked even after the modification of its size at $t=5.5 \mathrm{~s}$. Figs. 8 and 9 show the linear and angular velocities of the two followers, respectively. One can note that, when the leader turns back, robot $R_{3}$ accelerates (i.e., the linear velocity increases until $1 \mathrm{~m} / \mathrm{s}$ ), whereas robot $R_{2}$ slows down in order to keep the triangular shape.

\section{CONCLUSION}

In this paper, the formation control problem for wheeled mobile robots has been addressed. A robust second order slidingmode controller is proposed to control the leader-follower formation using only the measurement of relative configurations between robots. The controller does not need measurement or estimation of the leader velocity. It achieves the asymptotical stabilization of the vehicles toward a time-varying desired formation, in spite of the unavoidable presence of parametric uncertainties and disturbances, using the on board vision system. Furthermore, it ensures the collision avoidance from the initial time instance. Experimental results have demonstrated the effectiveness of the proposed strategy.

\section{REFERENCES}

[1] J. P. Desai, J. P. Ostrowski, and V. Kumar, "Modeling and control of formations of nonholonomic mobile robots," IEEE Trans. Robot. Autom., vol. 17, no. 6, pp. 905-908, Dec. 2001.

[2] P. Ogren, M. Egerstedt, and X. Hu, "A control Lyapunov function approach to multiagent coordination," IEEE Trans. Robot. Autom., vol. 18, no. 5 , pp. 847-851, Oct. 2002.
[3] T. Balch and R. Arkin, "Behavior-based formation control for multirobot teams," IEEE Trans. Robot. Autom., vol. 14, no. 6, pp. 926-939, Dec. 1998.

[4] J. Lawton, R. Beard, and B. Young, "A decentralized approach to formation maneuvers," IEEE Trans. Robot. Autom., vol. 19, no. 6, pp. 933-941, Dec. 2003.

[5] R. Beard, J. Lawton, and F. Hadaegh, "A coordination architecture for spacecraft formation control," IEEE Trans. Control Syst. Technol., vol. 9, no. 6, pp. 777-790, Nov. 2001.

[6] A. Das, R. Fierro, V. Kumar, J. P. Ostrowski, J. Spletzer, and C. Taylor, "A vision-based formation control framework," IEEE Trans. Robot. Autom., vol. 18, no. 5, pp. 813-825, Oct. 2002.

[7] S. Mastellone, D. M. Stipanovic, C. R. Graunke, K. A. Intlekofer, and M. W. Spong, "Formation control and collision avoidance for multi-agent non-holonomic systems: Theory and experiments," Int. J. Robot. Res., vol. 27 , no. 1 , pp. $107-126$, Jan. 2008.

[8] H. Takahashi, H. Nishi, and K. Ohnishi, "Autonomous decentralized control for formation of multiple mobile robots considering ability of robot," IEEE Trans. Ind. Electron., vol. 51, no. 6, pp. 1272-1279, Dec. 2004.

[9] R. Vidal, O. Shakernia, and S. Sastry, "Following the flock," IEEE Robot. Autom. Mag., vol. 11, no. 4, pp. 14-20, Dec. 2004.

[10] G. Mariottini, F. Morbidi, D. Prattichizzo, G. Pappas, and K. Daniilidis, "Leader-follower formations: Uncalibrated vision-based localization and control," in Proc. IEEE Conf. Robot. Autom., 2007, pp. 2403-2408.

[11] X. Li, J. Xiao, and Z. Cai, "Backstepping based multiple mobile robots formation control," in Proc. Conf. Intell. Robots Syst., 2005, pp. 887-892.

[12] J. Sanchez and R. Fierro, "Sliding mode control for robot formations," in Proc. IEEE Symp. Intell. Control, 2003, pp. 438-443.

[13] G. Mariottini, G. Pappas, D. Prattichizzo, and K. Daniilidis, "Vision based localization of leader-follower formations," in Proc. IEEE Conf. Decision Control, 2005, pp. 635-640.

[14] O. Orqueda and R. Fierro, "Robust vision-based nonlinear formation control," in Proc. Amer. Control Conf., 2006, pp. 1422-1427.

[15] V. I. Utkin and J. Shi, "Integral sliding mode in systems operating under uncertainty conditions," in Proc. IEEE Conf. Decision Control, 1996, pp. 4591-4596.

[16] M. Corradini and G. Orlando, "Control of mobile robots with uncertainties in the dynamical model: A discrete time sliding mode approach with experimental results," Control Eng. Pract., vol. 10, no. 1, pp. 23-34, Jan. 2002.

[17] M. Defoort, T. Floquet, A. Kökösy, and W. Perruquetti, "Integral sliding mode control for trajectory tracking of a unicycle type mobile robot," Integr. Comput.-Aided Eng., vol. 13, no. 3, pp. 277-288, Jul. 2006.

[18] M. Defoort, T. Floquet, A. Kökösy, and W. Perruquetti, "Decentralized robust control for multi-vehicle navigation," in Proc. Eur. Control Conf., 2007, pp. 2150-2157.

[19] S. V. Emel'yanov, S. V. Korovin, and L. V. Levantovskii, "Higher order sliding modes in the binary control systems," Sov. Phys. Dokl., vol. 31, no. 4, pp. 291-293, 1986.

[20] L. Fridman and A. Levant, "Higher order sliding mode modes," in Control in Engineering, E. W. Perruquetti and J. P. Barbot, Eds. New York: Marcel Dekker, 2002, pp. 53-102.

[21] A. Levant, "Robust exact differentiation via sliding mode technique," Automatica, vol. 34, no. 3, pp. 379-384, Mar. 1998. 
[22] V. I. Utkin, "Sliding mode control design principles and applications to electric drives," IEEE Trans. Ind. Electron., vol. 40, no. 1, pp. 23-36, Feb. 1993.

[23] A. Isidori, Nonlinear Control Systems, 3rd ed. ser. Communication and Control Engineering Series. New York: Springer-Verlag, 1995.

[24] G. Bartolini, A. Ferrara, and E. Usai, "Applications of a sub-optimal discontinuous control algorithm for uncertain second order systems," Int. J. Robust Nonlinear Control, vol. 7, no. 4, pp. 299-319, Apr. 1998

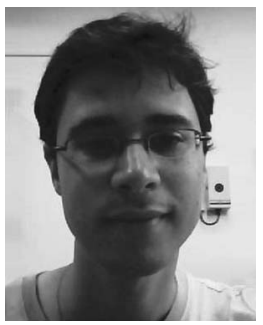

Michael Defoort was born in France in 1981. He received the M.S. degree in automatic control from the Institut Supérieur d'Electronique et du Numérique, Lille, France, in 2004, and the Ph.D. degree from the Ecole Centrale de Lille, Lille, in 2007.

Since 2007, he has been with the Department of System Design Engineering, Keio University, Yokohama, Japan, where he is a Visiting Researcher. His research interests include nonholonomic systems, nonlinear control, motion planning, and formation control.

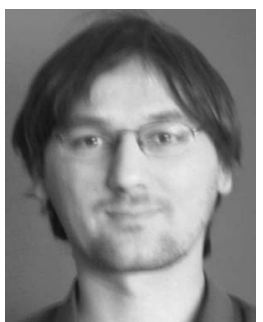

Thierry Floquet received the M.S. and Ph.D. degrees in automatic control from the University of Lille, Lille, France, in 1997 and 2000, respectively.

Since 2001, he has been with the Laboratoire d'Automatique, Génie Informatique et Signal, Unité Mixte de Recherche 8146, Centre National de la Recherche Scientifique, Ecole Centrale de Lille, Villeneuve-d'Ascq, France, where he is Chargé de Recherche of the Centre National de la Recherche Scientifique. His research interests include observer theory and nonlinear control with applications to electrical engineering and robotics.

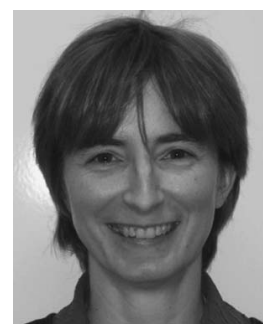

Annemarie Kökösy was born in Romania in 1970 She received the Diploma in automatic control engineering from the University "Politehnica" Timisoara, Timisoara, Romania, in 1994, the M.S. degree from the Ecole Nationale d'Ingénieurs de Belfort, Belfort, France, in 1995, and the Ph.D. degree from the University Franche-Comté, Besançon, France, in 1999.

From 1998 to 1999, she was an Assistant Professor with the Department of Automatic Control, University Franche-Comté. From 1999 to 2000, she was an Assistant Professor with the Department of Automatic Control, University of Lille, Lille, France. Since May 2000, she has been with the Department of Signals and Systems, Institut Supérieur d'Electronique et du Numérique, Lille, France, where she is currently an Assistant Professor. Since 2003, she has been the Head of the Department of Signals and Systems. She is also with the Laboratoire d'Automatique, Génie Informatique et Signal, Unité Mixte de Recherche 8146, Centre National de la Recherche Scientifique, Ecole Centrale de Lille. Her research interests include robotics and analysis and control for nonlinear systems.

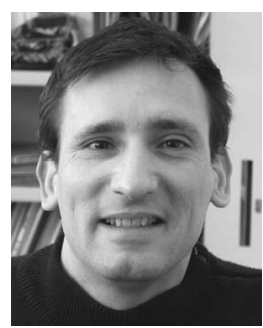

Wilfrid Perruquetti was born in Saint Gilles, France, in 1968. He received the M.Sc. degree in automatic control from the "Institut Industriel du Nord" (French "Grande Ecole"), Lille, France, in 1991. He obtained the Ph.D. degree in automatic control in 1994.

He was with the "Ecole Centrale de Lille" (French "Grande Ecole"), Lille, as an Assistant Professor in 1995, where he has been a Full Professor since 2003 and is working in the Laboratoire d'Automatique, Génie Informatique et Signal, Unité Mixte de Recherche 8146, Centre National de la Recherche Scientifique and within the project ALIEN of INRIA-Lille Nord Europe. He has published about 128 book chapters, journal, and conference papers and is the Coeditor of two books with Jean-Pierre Barbot: Sliding Mode Control in Engineering (Marcel Dekker) and Chaos in Automatic Control (CRC-Taylor and Francis). His research interests include stability analysis, (finite-time) stabilization (in particular, stabilization), sliding-mode control of nonlinear and time-delay systems, and observation and identification of system parameters. His main fields of applications are in robotics (mobile robots and robotic manipulators) and electrical actuators (dc, induction, and stepper motors).

Prof. Perruquetti has been a member of two International Federation of Automatic Control Technical Committees (2.3 "Nonlinear Control Systems" and 2.5 "Robust Control") since 2002, and he is a Senior Member of the Société de l'Electricité, de l'Electronique et des Technologies de l'Information et de la Communication. In 2007, he was appointed as a representative of the Direction Générale de la Recherche et de l'Innovation by the French Ministry of Education and Research. He was the Program Director of several programs and was involved in several International Program Committees. He is currently the Editor-in-Chief of e-revue Sciences et Technologie de l'Automatique. 